\title{
Why precision medicine should be applied across the continuum of care for metastatic colorectal cancer patients
}

\author{
Marco Puzzoni ${ }^{1}$, Pina Ziranu ${ }^{1}$, Laura Demurtas ${ }^{1}$, Eleonora Lai ${ }^{1}$, Stefano Mariani ${ }^{1}$, Nicole \\ Liscia ${ }^{1}$, Paolo Soro ${ }^{1}$, Andrea Pretta ${ }^{1}$, Valentino Impera ${ }^{1}$, Silvia Camera ${ }^{1}$, Francesca Musio ${ }^{1}$, \\ Mara Persano ${ }^{1}$, Clelia Donisi ${ }^{1}$, Simona Tolu${ }^{1}$, Francesca Balconi ${ }^{1}$ \& Mario Scartozzi*, 1 \\ ${ }^{1}$ Medical Oncology Unit, University Hospital \& University of Cagliari, Cagliari, Italy \\ *Author for correspondence: Tel.: +39 0705109 3217; Fax: +39 0705109 3215; marioscartozzi@gmail.com
}

First draft submitted: 7 October 2019; Accepted for publication: 19 November 2019; Published online: 3 December 2019

Metastatic colorectal cancer (mCRC) treatment has been improved by several effective therapies, including chemotherapeutic agents combined with monoclonal antibodies targeting the EGFR or the VEGF. The multikinase inhibitor regorafenib and the fluoropyrimidine trifluridine/tipiracil are indicated for pretreated patients. However, despite the efforts in clinical and translational research, no novel drugs have recently been included in the $\mathrm{mCRC}$ therapeutic scenario and the results obtained in pretreated patients are disappointing. Therefore, we have to improve the management of the already available treatment options in order to adapt them to the dynamic nature of tumor biology and to the genetic heterogeneity of colorectal cancer. In this respect, the global treatment strategy would not be obtusely based only on $R A S-B R A F$ status on tissue samples at diagnosis but also on the basis of the recent findings regarding the pulsatile behavior of $R A S$ clones under EGFR blockade.

Precision medicine is well established in terms of $R A S$ status for first-line decision-making in mCRC. To date, we are able to exclude from anti-EGFR therapy putative refractory patients with $R A S$-mutated profile evaluated on tissue sample at diagnosis; therefore, positive predictive factors are lacking. Moreover, many other molecular factors are probably involved in primary resistance to anti-EGFR therapy. The mutational status of $B R A F$ is one of the most utilized predictive factors for $\mathrm{mCRC}$ in clinical practice; nevertheless, its predictive role remains unclear [1].

Furthermore, EGFR gene copy number, PIK3CA mutations, PTEN mutations and ERBB2 amplification are among the most important putative predictive determinants for anti-EGFR therapy, although not included into clinical practice [2-4].

In addition to anti-EGFR therapy primary resistance, the secondary resistance represents a challenging issue in mCRC treatment and it is closely related to the dynamic nature of tumor biology and to the colorectal cancer genetic heterogeneity. It is known, in fact, that $R A S$ status is not fixed and it is dynamic over time. The mechanism underlying the acquired resistance to the anti-EGFR, although not yet fully understood, seems to be linked with the emergence of EGFR downstream pathway mutation. In particular, the occurrence of $K R A S$ mutation is a mediator of acquired resistance to EGFR blockade and this mutation can be detected with circulating, cell-free DNA analysis (liquid biopsy) [5]. In this connection, the results from the study of Siravegna et al. in 2015 are particularly important [6]: the authors observed that KRAS mutant alleles emerging at the time of disease progression to antiEGFR based therapy declined in blood during anti-EGFR antibody suspension. The pulsatile behavior of $R A S$ clones under EGFR blockade [7] has acquired an increasing importance in the precision oncology over the last few years. This is partly due to the diffusion of the liquid biopsy, which represents a useful tool for monitoring dynamic $R A S$ status. On the other hand, the results of recent studies regarding rechallenge with anti-EGFR and the anti-EGFR treatment in patients who have a change in $R A S$ status (neo-RAS WT) has triggered further research development.

There are several studies which specifically assess the role of rechallenge with anti-EGFR in patients with mCRC. Santini et al. [8] investigated the activity of retreatment with a cetuximab-based therapy in 2012, achieving encouraging results in terms of outcome: the objective response rate was $53.8 \%$, the median progression free survival was 6.6 months. Siena et al. [9] retrospectively collected data from patients with RAS WT colorectal cancer enrolled in two randomized controlled trials (i.e., PRIME and PEAK) receiving panitumumab who were rechallenged with

Future $\because$ Medicine 
an EGFR inhibitor. Globally, 69/675 patients (10\%) were rechallenged with an EGFR inhibitor; median overall survival after rechallenge was 14.2 months (10.2-17.7). Cremolini et al. [10] designed a prospective Phase II trial of rechallenge with cetuximab plus irinotecan (i.e., CRICKET).

This prospective study evaluated patients with $R A S-B R A F$ wild type status, verified on tissue sample, who received irinotecan plus cetuximab-based regimen as first-line treatment. They underwent retreatment with cetuximab plus irinotecan. In addition, liquid biopsies for ctDNA were performed at baseline. RAS mutations were found in $48 \%$ $(12 / 25)$ patients. As for primary end point, response rate was $21 \%$; $95 \%$ CI: $10 \%-40 \%$. Notably, all patients achieving a partial response were $R A S$ wild-type in the ctDNA. No RAS mutations were detected in samples from patients who achieved confirmed partial response. Similarly, Siena et al. designed the CHRONOS trial (NCT03227926) [11] to assess the efficacy of rechallenge with panitumumab in patients not harboring any drivers of resistance to EGFR inhibitors on tissue samples, experiencing a $>50 \%$ drop in ctDNA RAS levels.

Beyond the concept of the pulsatile behavior of $R A S$ clones under EGFR blockade, recent studies suggested that $R A S$-mutated mCRC may convert to wild type status (neo- $R A S$ wild type) [12,13]. Moreover, recent data showed benefit of cetuximab in patients with neo-RAS wt mCRC. Raimondi et al. [12] prospectively evaluated 11 patients with mCRC-defined $R A S$ mutant on primary tumor biopsy. The $R A S$ mutational status was assessed at progression to anti-VEGF based treatments. A real-time PCR was used to screen $R A S$ mutational profile from plasma. Plasma samples with $R A S$ wt profile at real-time PCR were subsequently analyzed with next-generation sequencing (NGS). $R A S$ wild type status was found in four patients $(4 / 11,36 \%)$ whereas, in six patients $(6 / 11), R A S$-mutant profile was confirmed. Discordant real-time PCR and next-generation sequencing results were found in $1 / 11$ patients. The population with de novo RAS type status were considered eligible to anti-EGFR treatment and they were treated accordingly, obtaining promising results.

In conclusion, given the dynamic nature of tumor biology and the genetic heterogeneity of colorectal cancer, the simple evaluation of $R A S-B R A F$ status on tissue samples at diagnosis is not sufficient. A growing understanding of tumor biology enables precision medicine to be applied across lines of therapy and liquid biopsy represents a useful tool for monitoring $R A S$ status. Nevertheless, the reduced sample size of the mentioned Phase II prospective trial regarding the rechallenge with anti-EGFR prevents us from deriving a definitive conclusion. On the other hand, recent findings about anti-EGFR treatment in patients with neo- $R A S$ wt tumor profile lend strong support to this challenging approach. Turning into clinical practice the rechallenge strategy with anti-EGFR could be a viable therapeutic option in pretreated patients meeting the inclusion criteria of CRICKET trial. Results from CHRONOS trial are expected to give new insight in this challenging issue. As for anti-EGFR treatment in neo-RAS wt, in the face of the strong biological rationale, the actual scientific evidence is not enough to be translated yet into clinical practice.

\section{Author contributions}

Marco Puzzoni, Pina Ziranu, Laura Demurtas, Eleonora Lai, Stefano Mariani, Nicole Liscia, Paolo Soro, Andrea Pretta, Valentino Impera, Silvia Camera, Mara Persano, Clelia Donisi, Simona Tolu, Francesca Balconi and Mario Scartozzi performed conception, writing and revision of the manuscript. All authors read and approved the final manuscript.

Financial \& competing interests disclosure

The authors have no relevant affiliations or financial involvement with any organization or entity with a financial interest in or financial conflict with the subject matter or materials discussed in the manuscript.

No writing assistance was utilized in the production of the manuscript.

\section{References}

1. Scartozzi M, Giampieri R, Aprile G et al. The distinctive molecular, pathological and clinical characteristics of BRAF-mutant colorectal tumors. Expert Rev. Mol. Diagn. 15(8), 979-987 (2015).

2. Giampieri R, Mandolesi A, Abouelkhair KM et al. Prospective study of a molecular selection profile for RAS wild type colorectal cancer patients receiving irinotecan-cetuximab. J. Transl. Med. 13(140), 1 (2015).

3. Yang ZY, Wu XY, Huang YF et al. Promising biomarkers for predicting the outcomes of patients with KRAS wild-type metastatic colorectal cancer treated with anti-epidermal growth factor receptor monoclonal antibodies: a systematic review with meta-analysis. Int. J. Cancer 133(8), 1914-1925 (2013).

4. Sartore-Bianchi A, Amatu A, Porcu L et al. HER2 positivity predicts unresponsiveness to EGFR-targeted treatment in metastatic colorectal cancer. Oncologist 24(10), 1395-1402 (2019). 
5. Diaz LA Jr, Williams R, Wu J et al. The molecular evolution of acquired resistance to targeted EGFR blockade in colorectal cancers. Nature 486(7404), 537-540 (2012).

6. Siravegna G, Mussolin B, Buscarino M et al. Monitoring clonal evolution and resistance to EGFR blockade in the blood of metastatic colorectal cancer patients. Nat. Med. 21(7), 795-801 (2015).

7. Mauri G, Pizzutilo EG, Amatu A et al. Retreatment with anti-EGFR monoclonal antibodies in metastatic colorectal cancer: systematic review of different strategies. Cancer Treat. Rev. 73, 41-53 (2019).

8. Santini D, Vincenzi B, Addeo R et al. Cetuximab rechallenge in metastatic colorectal cancer patients: how to come away from acquired resistance? Ann. Oncol. 23, 2313-2318 (2012).

9. Siena S, George P, Gerald P et al. Rechallenge with EGFR inhibitors in patients with metastatic colorectal cancer: effect on out-comes. Ann. Oncol. 28, 113 (2017).

10. Cremolini C, Rossini D, E Dell'Aquila E et al. Rechallenge for patients with RAS and BRAF wild-type metastatic colorectal cancer with acquired resistance to first-line cetuximab and irinotecan: a Phase II single-arm clinical trial. JAMA Oncol. 5(3), 343-350 (2019).

11. Siena S, Bardelli A, Sartore-Bianchi A et al. Exploiting clonal evolution and liquid biopsy to overcome resistance to anti-EGFR treatment in metastatic colorectal cancer: the CHRONOS trial. Abstracts: AACR-NCI-EORTC International Conference. Mol. Targets Cancer Ther. 17(Suppl. 1), Abstract A089 26-30 (2017).

12. Raimondi C, Nicolazzo C, Belardinilli F et al. Transient disappearance of RAS mutant clones in plasma: a counterintuitive clinical use of EGFR inhibitors in RAS mutant metastatic colorectal cancer. Cancers (Basel) 11(1), 42 (2019).

13. Sunakawa $Y$, Usher J, Satake $\mathrm{H}$ et al. Gene mutation status in circulating tumor DNA (ctDNA) and first-line FOLFOXIRI plus bevacizumab (bev) in metastatic colorectal cancer (mCRC) harboring RAS mutation. Ann. Oncol. 29(Suppl.8), viii150-viii204 (2018). 
